\title{
Improving Public Health Preparedness: Strengthening Biosurveillance Systems for Enhanced Situational Awareness
}

\author{
Nadja Vielot* \\ UNC Gillings School of Global Public Health, Chapel Hill, NC, USA
}

\section{Objective}

To review and summarize best practices of thought leaders and implementers of biosurveillance systems with an emphasis on event-related situational awareness.

\section{Introduction}

In emergencies, public health agencies must be able to respond to health threats that can affect entire communities. Agency leaders need to achieve situational awareness through the development of flexible, timely, and accurate electronic biosurveillance systems. Drawing on various sources, the North Carolina Preparedness and Emergency Response Research Center (NCPERRC) and Public Health Informatics Institute (PHII) developed recommendations for state and local public health agencies to build or enhance their biosurveillance capabilities.

\section{Methods}

We searched academic literature and white papers to determine what biosurveillance systems are in place around the country and how they function. We also conducted key informant interviews with biosurveillance experts to gain their insights into how best to conduct biosurveillance to improve situational awareness and their recommendations on key components necessary for systems to operate. Next, we applied PHII's Collaborative Requirements Development Methodology (CRDM) to identify core business processes and tasks involved in biosurveillance. A workgroup of biosurveillance experts then refined the business processes and tasks. They also developed a set of priority data sources, along with guiding principles to inform system development and improvement. Case studies on the financial and personnel resources necessary to sustain a system are in progress.

\section{Results}

Notifiable disease reports, emergency department data, and electronic laboratory reports are the primary data sources that inform biosurveillance systems, given their availability and timeliness in an electronic environment ${ }^{1-3}$. However, other sources may be useful in emergencies. Since jurisdictions have varying information needs and often customize their systems, we developed guidance to develop systems with minimum capabilities for effective biosurveillance:

Systems used during emergencies should be based on those used for routine surveillance.

$\bigcirc$ Systems require ongoing funding and adequate staffing for optimization as new technology and needs develop.

$\bigcirc$ Systems must support workflow at the local and state levels, and provide local, state, regional and national situational awareness during an event.

$\bigcirc$ Systems require formal analysis of public health business processes and workflow when developing requirements to guide their design and development.

Systems must comply with applicable standards in order to be scalable and interoperable.

Systems must adapt to local needs, particularly in the context of event response where customization may be needed.
Adhering to these principles will ensure that jurisdictions achieve effective routine biosurveillance, with potential for more sophisticated operations. For example, the Georgia SendSS system was modified remotely to monitor health status in shelters after Hurricane Katrina, and Florida's ESSENCE system was customized to detect bioterrorism events during the Republican National Convention in 2012.

\section{Conclusions}

Biosurveillance must function within an all-hazards model and should be flexible enough to respond to a wide array of needs. Systems must be technologically capable of receiving and processing health data to detect health threats and must be staffed and financed adequately to maintain systems functions. In response to varying biosurveillance needs, we identified the core capabilities that systems must have to allow them to be customizable and the most useful to protect health during emergencies.

\section{Keywords}

biosurveillance; preparedness; situational awareness; capabilities; business process analysis

\section{Acknowledgments}

Thanks to Ed Baker and Jessica Southwell of UNC; David Ross, Vivian Singletary, LaToya Osmani, Juneka Rembert, and Katie Nolen of PHII; all surveillance experts who contributed their insights; and Perry Smith for comments on this abstract.

\section{References}

1. Fangman M, Samoff E, Waller A. Electronic Laboratory Reporting Improves Surveillance Processing at Local and State Health Departments [Internet]. Carrboro, North Carolina; 2012. Available from: http:// cphp.sph.unc.edu/ncperrc/research/elec_lab_reporting.pdf

2. Travers D, Barnett $\mathrm{C}$, Ising A, Waller A. Timeliness of Emergency Department Diagnoses for Syndromic Surveillance. AMIA 2006 Symposium Proceedings [Internet]. 2006. p. 769-73. Available from: http://www.pubmedcentral.nih.gov/articlerender.fcgi?artid $=1839358 \&$ tool $=$ pmcentrez\&rendertype $=$ abstract

3. Centers for Disease Control and Prevention. CDC's Vision for Public Health Surveillance in the 21 st Century. MMWR Morbidity and Mortality Weekly Report. 2012.

\section{*Nadja Vielot}

E-mail: nadjavielot@unc.edu 